\title{
En el Infarto agudo al miocardio los niveles plasmáticos de microvesículas extracelulares se elevan más precozmente que el aumento de la Troponina-I
}

\author{
Daniel Hevia ${ }^{1 a}$, Jorge Cifuentes ${ }^{1 b}$, Olga Alvarado ${ }^{1 c}$, Pamela San Martín ${ }^{1 d}$, Pía Venegas ${ }^{1 e}$, Michel Coron ${ }^{2}$, \\ Ignacio Cabrera ${ }^{2}$, Sofía López ${ }^{1 f}$, Karin Oyarce ${ }^{1 f}$, Francisca Rojas ${ }^{1 f,}$ Ximena Sazo $^{1 f}$, Martina Vallejos ${ }^{1 f,}$ Tania Weil ${ }^{1 f \text {, }}$ \\ Ricardo Larrea $^{2}$, Carlos E. Irarrázabal ${ }^{1 a}$.
}

\author{
1 Laboratorio de Fisiología Integrativa y Molecular, Facultad de Medicina, Universidad de los Andes, Santiago-Chile. \\ 2 Departamento de Enfermedades Cardiovasculares, Clínica Dávila. \\ a PhD, Ciencias Biomédicas. \\ b Bioquímico. \\ c Técnico en Laboratorio Clínico y Banco de Sangre. \\ $d$ Estadístico asociado al proyecto. \\ e Enfermera. \\ f Estudiante de Medicina Universidad de los Andes, Santiago-Chile \\ Trabajo realizado en Laboratorio de Fisiología Integrativa y Molecular, Facultad de Medicina, Universidad de los Andes, \\ Santiago-Chile y en colaboración con Departamento de Enfermedades Cardiovasculares, Clínica Dávila. \\ Financiado por proyecto Corfo 14IDL2-30168.
}

Introducción: La Troponina I (TnI) plasmática es el biomarcador "Gold" estándar utilizado en diagnóstico de Infarto Agudo al Miocardio (IAM), indicando necrosis cardíaca. Las microvesículas extracelulares (MVEC), participan en comunicación celular, por lo que estudiar su distribución entregaría información respecto del evento isquémico, antesala del infarto.

Objetivo: Estudiar las MVECs plasmáticas en pacientes con Síndrome Coronario Agudo (SCA) y compararlas con los niveles de TnI.

Métodos: Plasma de 22 pacientes controles se recolectó 0 -2hrs post-ingreso a urgencia. Plasma de 45 pacientes SCA se recolectó 0-2, 6-8 y 10-14hrs post ingreso, junto con la toma de muestra para estudio de TnI. Las MVECs plasmáticas fueron enriquecidas mediante kit comercial. La determinación de la concentración y tamaño MVECs se realizó por NTA (Nanoparticles Tracking Assay) usando el equipo Nanosight.
Resultados: La concentración promedio de MVECs 0-2 hrs post ingreso fue 7,2 veces superior en plasma de pacientes con SCA vs controles y la moda del tamaño disminuyó en pacientes con SCA. La TnI no mostró diferencias significativas en 0-2 hrs post ingreso en el grupo estudiado. La concentración de las MVEC disminuyó significativamente después de 10-14 hrs post ingreso, mientras que la concentración promedio TnI se mantuvo invariable demostrando el aumento de MVECs previo al incremento de TnI.

Conclusión. El aumento de MVECs previo al incremento de la TnI en pacientes infartados, sugiere que las MVECs aumentan en la fase previa del IAM, como respuesta al daño tisular. Actualmente, estudiamos el contenido molecular de las MVECs, para establecer un método diagnóstico del Síndrome Coronario Agudo basado en MVECs.

Financiado por proyecto Corfo 14IDL2-30168.

Correspondencia

Dr. Carlos Irarrázabal.

Av. Plaza 2501, Las Condes, Santiago-Chile.

cirarrazabal@uandes.cl. 


\section{Plasma levels of extracellular macrovesicles are elevated earlier than troponin-I in patients with myocardial infarction}

Background: Troponin I (TnI) is the gold standard used to establish the diagnosis of myocardial infarction (AMI), indicating the presence of myocardial necrosis. Extracellular micro vesicles are involved in cellular communication. Their distribution may provide information relating to the development of AMI in patients with acute coronary syndromes (ACS)

Aim: to study plasma levels of ECMV compared to those of TnI in patients with ACS.

Methods: The plasma levels of TnI and ECMV from 22 control patients coming to the emergency units was compared to plasma from 45 patients with ACS. Levels of both parameters were determined 0-2, 6-8 and 10-14 hours post admission. ECMVs were enriched by means of a commercial kit. Concentration and size of ECMV was determined by
NTA (Nanoparticles tracking assay) using the Nanosight equipment.

Results: Plasma concentration of ECMV was 7.2 times higher than that of TnI 0-2 hrs post admission. The mode of ECMV size was lower in patients with ACS. Concentration of ECMV had decreased significantly 10-14 hrs post admission, whereas the TnI levees remained stable.

Conclusion: The increase in ECMV earlier than TnI in AMI suggests that ECMV are elevated in the pre-AMI phase, as a response to early tissue damage. A study of cellular content of ECMV, being carried out, may lead to develop a method for the early diagnosis of AMI in patients with ACS.

Key words: Acute coronary syndrome; acute myocardial infarction; troponin-I; extracellular micro vesicles. 


\section{Introducción:}

Durante el año 2013, 1 de cada 3 muertes fue originada por enfermedad cardiovascular (ECV). Según la Organización Mundial de la Salud (OMS), la ECV es la primera causa de mortalidad en el mundo, llegando a 17,5 millones de muertes durante el año 2012, de los cuales $38 \%$ se debió a patología coronaria ${ }^{1}$.

También en Chile el infarto agudo del miocardio (IAM) corresponde a la primera causa de muerte, con un total de 5.895 fallecidos (tasa de 36 por 100.000 habitantes) y 7.942 egresos hospitalarios durante el $2006^{2}$. Durante la década del 2000-2010 la muerte asociada a falla cardíaca se priorizó en los objetivos sanitarios de nuestro país, cuya meta específica era reducir en un 30\%, la mortalidad asociada a esta patología. Aunque los esfuerzos han permitido una significativa reducción de la mortalidad, no se ha logrado el objetivo planteado ${ }^{2}$.

La isquemia cardíaca suele manifestarse con signos clínicos repentinos, en el llamado Síndrome Coronario Agudo (SCA) ${ }^{3}$, y también puede ocurrir un estado de isquemia cardíaca crónica clínica o silente. En este último caso, los pacientes tienen elevado riesgo de experimentar una muerte súbita o un IAM.

Frente a la sospecha de un IAM, es fundamental realizar la confirmación diagnóstica precoz, ya que la evolución del daño es rápidamente progresiva. La necrosis miocárdica se inicia a los 20 a 30 min de la oclusión de la arteria coronaria en la región subendocárdica y se extiende en forma progresiva en sentido externo hacia la zona subepicárdica. Así, en un período de 3 hrs, la necrosis compromete al $75 \%$ de la pared del miocardio y se completa después de las primeras 6 hrs de evolución. En este contexto, el pronóstico precoz del paciente es determinante, de manera que el inicio rápido del tratamiento permitirá salvar mayor cantidad de miocardio viable.

Hasta el momento, el análisis del contenido plasmático de Troponina y Creatina-quinasa (CK y CK-MB) es el mejor y más usado indicador bioquímico de $\mathrm{IAM}^{4,5}$. La elevación en los niveles de estas proteínas indica que ha ocurrido un IAM, mientras que un posterior descenso es indicativo que el IAM ha terminado. Estos marcadores son liberados durante la destrucción del cardiomiocito y no se elevan antes de tres o cuatro horas de iniciado el infarto (por ello no permiten su detección precoz) y, además, no son totalmente exclusivos de este cuadro ${ }^{6}$. Esta evidencia permite determinar que necesitamos mejores biomarcadores para evaluar el riesgo de esta enfermedad. Los exosomas son un tipo de microvesículas extracelulares (MVECs) de 30-100 nm, secretadas por las células, que contienen material genético y proteínas, los cuales reflejan cambios metabólicos que experimenta una célula en un momento determinado ${ }^{7-13}$. Estos MVEC/ exosomas pueden circular en el plasma y la función que cumplen en el corazón, es aún un campo en desarrollo ${ }^{24}$. Sin embargo, la hipótesis que se ha propuesto es que los exosomas actuarían como centinelas y estarían preparados para participar en el remodelamiento de tejido como respuesta a la alteración o destrucción de la matríz extracelular; activando las vías de cardioprotección del IAM $^{11}$. La obtención de estas MVECs permitiría el estudio directo de marcadores que fueron sintetizados en la célula original expuesta al daño, antes que ocurra la necrosis celular.

En la presente investigación, exploramos la hipótesis que las MVECs del tipo exosomas participan en la respuesta a condiciones del SCA. Estudios in vitro realizados por Gupta y Knowlton, indicaron que en el medio de cultivo proveniente de cardiomiocitos de rata, sometidos a hipoxia/reoxigenación, la proteína del shock térmico Hsp60, fue secretada en exosomas ${ }^{14}$. De este modo, se establece un mecanismo mediante el cual los cardiomiocitos responden a señales de peligro/daño a través de la liberación de exosomas. Es por ello que el objetivo de nuestro estudio radica en caracterizar las MVECs/exosomas en plasma de pacientes con IAM y compararlas con los niveles de TnI. La elevación más precoz de MVEC/ Exosomas permitiría identificar los pacientes con SCA y por tanto evaluar el riesgo de IAM.

\section{Métodos}

\section{Estudio clínico y reclutamiento de pacientes}

El estudio fue aprobado por el Comité Ético-Científico de la Clínica Dávila (10082015). Todos los pacientes firmaron consentimiento informado. En forma prospectiva se recolectó muestras de 67 pacientes durante septiembre del 2015 y junio del 2016. Los criterios de inclusión fueron los siguientes: mayores de 18 años, firma de consentimiento informado y no poseer antecedentes de enfermedades cardíacas previas en el caso de los controles. Se logró obtener muestras de 22 pacientes de Urgencia sin diagnóstico de SCA (Control) y 45 pacientes con SCA. De cada paciente se obtuvieron muestras de sangre con anticoagulante (EDTA). En el caso de pacientes con SCA, la toma de muestra se realizó en el mismo momento que para el estudio de TnI plasmática en un curso temporal de $24 \mathrm{~h}, 0-2 \mathrm{~h}$ después del ingreso (primera muestra), 6-8 h (segunda muestra) y 10-14 h (tercera muestra) post ingreso. El plasma fue almacenado a $-80^{\circ} \mathrm{C}$. 


\section{Obtención de MVEC.}

El plasma fue descongelado y centrifugado a $2100 \mathrm{~g}$ por 15 min a temperatura ambiente. Se recuperó el sobrenadante y se filtró por un poro de $0,22 \mu \mathrm{m}$ (Millipore). De esta fracción filtrada se recolectaron $250 \mu \mathrm{L}$ y se centrifugaron a $3100 \mathrm{~g}$ durante $15 \mathrm{~min}$ a $4^{\circ} \mathrm{C}$. El sobrenadante se mezcló con $63 \mu \mathrm{L}$ de ExoQuick ${ }^{\circledR}$ y se incubó toda la noche a $4^{\circ} \mathrm{C}$. Luego de centrifugar a $1500 \mathrm{~g}$ por $30 \mathrm{~min}$ a $4^{\circ} \mathrm{C}$, se descartó el sobrenadante y el pellet fue re-suspendido en $80 \mu \mathrm{L}$ de PBS $1 \mathrm{X}$ (MVEC-ExoQ). El homogenizado de MVEC se almacenó a $-80^{\circ} \mathrm{C}$ hasta su uso. En el caso de ultracentrifugación se utilizaron $30 \mathrm{~mL}$ de plasma de un paciente sano y se sometieron a $2000 \mathrm{~g}$ por $30 \mathrm{~min}$ a $4^{\circ} \mathrm{C}$. Se recuperó el sobrenadante y se centrifugó a $12000 \mathrm{~g}$ por $45 \mathrm{~min}$ a $4^{\circ} \mathrm{C}$. El sobrenadante se centrifugó a $110000 \mathrm{~g}$ por $120 \mathrm{~min}$ a $4^{\circ} \mathrm{C}$. Se descartó el sobrenadante, se resuspendió el pellet en PBS 1X y se centrifugó a $110000 \mathrm{~g}$ por $70 \mathrm{~min}$ a $4^{\circ} \mathrm{C}$. Finalmente, cada pellet resultante se resuspendió en $50 \mu \mathrm{L}$ de PBS $1 \mathrm{X}$ y se almacenó a $-80^{\circ} \mathrm{C}$ hasta su uso.

\section{Análisis de tamaño y concentración de MVEC}

El análisis de MVEC se realizó con la tecnología " $\mathrm{Na}$ noparticles Tracking Assay" (NTA), utilizando el equipo Nanosight LM ${ }^{20}$ (Malvern Instruments). Las MVEC fueron analizadas desde plasma y de una fracción enriquecida de MVEC (MVEC-ExoQ) mediante el kit comercial ExoQuick®, de acuerdo a lo expuesto previamente ${ }^{8,19}$. Los controles del análisis de las MVEC incluyeron un control negativo consistente de PBS. Además, se utilizó una solución de partículas de Látex-Poliestireno (Malvern Instruments, Cat. No.: NTA4088) de $100 \mathrm{~nm}$ de tamaño como control positivo de la identificación de MVEC por Nanosight. Por cada muestra se analizaron $>200$ trazados (tracks) y se grabaron al menos dos videos de 60 seg cada uno.

\section{Detección de TnI.}

Análisis de troponina-I plasmática se realizó como se menciona en Loh et al, 2016 25 ; empleando un equipo Vitros 5600 (Ortho Clinical Diagnostics); Western Blot; A partir de la solución de MVEC-ExoQ obtenida mediante ExoQuick $\AA$, se tomaron $40 \mu \mathrm{L}$ y se mezclaron con $40 \mu \mathrm{L}$ de Buffer de lisis comercial (Roche). La concentración de proteínas fue determinada utilizando el kit comercial Micro-BCA (Thermo Scientific), de acuerdo a lo descrito por el fabricante. Para la medición de las tetraspaninas se realizó un gel de electroforesis denaturante (SDS-PAGE) con $50 \mu \mathrm{g}$ de proteínas provenientes de MVEC-ExoQ y $15 \mu \mathrm{g}$ de proteínas provenientes de ultracentrifugación. Una vez finalizada la electroforesis, las proteínas fueron transferidas a una membrana de nitrocelulosa (Thermo Scientific) para luego ser bloqueadas durante $1 \mathrm{~h}$ con mezcla de PBS-Buffer OD (Li-Cor; 1:1) a temperatura ambiente. Anticuerpos primarios contra CD9 (Abcam) y CD63 (Santa Cruz) fueron empleados 1:1000 en solución de bloqueo. Un anticuerpo secundario conjugado a fluoróforo infra rojo (IR) fue empleado 1:15000 en solución de bloqueo y la emisión de fluorescencia fue detectada con equipo Odyssey CLx (Li-Cor) por fluorescencia IR.

\section{Análisis estadístico}

Los datos se muestran como valores promedios \pm desviación estándar. Las variables continuas fueron descritas como promedio y desviación estándar y comparadas mediante la prueba t-Student y en caso de comparación de más de 2 grupos se utilizó ANOVA.

\section{Resultados}

\section{Caracterización de la población de pacientes estu- diados}

Las muestras analizadas corresponden a pacientes que ingresaron a la Unidad de Urgencia y a la Unidad de Cardiología de la Clínica Dávila. La Tabla 1 muestra los datos demográficos para 22 pacientes de urgencia (Control) y 45 SCA (Síndrome Coronario Agudo). Los individuos reclutados fueron hombres en el $41 \%$ del grupo control $y$ en el $62 \%$ del grupo con SCA. La edad promedio fue $58,3 \pm 17,6$ en controles y $62,5 \pm 11,6$ en SCA. Los pacientes controles poseen niveles de $\mathrm{TnI}<0.012 \mathrm{ng} / \mathrm{mL}$, mientras que los SCA poseen niveles de TnI que van desde $0.013-395 \mathrm{ng} / \mathrm{mL}$ durante las primeras $24 \mathrm{~h}$ de ingreso a la Clínica. En el grupo de SCA el 13,3\% (6/45) fue diagnosticado como angina inestable y el 66,7\% (30/45) como IAM y el resto con otra patología cardíaca. En el grupo con SCA se presentó un mayor porcentaje de Diabetes Mellitus (40\%), hipertensión arterial $(75,6 \%)$, dislipidemia $(66,7 \%)$, tabaquismo $(42,2 \%)$ y antecedentes de previos de IAM (11\%), que en los controles (Tabla 1).

\section{Pacientes SCA presentaron un incremento de las MVEC/Exosomas presentes en plasma humano}

Se realizó un enriquecimiento de MVEC/Exosomas a partir de plasma filtrado (poro de $0,22 \mu \mathrm{m}$ ) mediante ExoQuick®. Estudios preliminares realizados por nuestro grupo permitieron establecer que el kit comercial muestra una muy buena reproducibilidad en la purifica- 
Tabla 1. Características de los pacientes en estudio.

\begin{tabular}{lcc} 
& Control & SCA \\
& 22 & 45 \\
\hline Sexo (Género= n; \%) & Masculino $=9 ; 41 \%$ & Masculino $=28,62 \%$ \\
\hline Edad (Promedio + ED) & $58,3 \pm 17,6$ & $62,5 \pm 11,6$ \\
\hline & $\mathrm{Al}=0$ & $\mathrm{Al}=6 ; 13,3 \%$ \\
& $\mathrm{IAM}=0$ & $\mathrm{IAM}=30 ; 66,7 \%$ \\
Diagnóstico (n; \%) & Otra $=0$ & Otra $=9 ; 20 \%$ \\
& & \\
\hline Tnl (Rango, ng/mL) & $<0.012 \mathrm{ng} / \mathrm{mL}$ & $0.013-395 \mathrm{ng} / \mathrm{mL}$ \\
\hline Diabetes (n; \%) & $3 ; 13,6 \%$ & $18 ; 40 \%$ \\
\hline Hipertensión (n; \%) & $9 ; 40,9 \%$ & $34 ; 75,6 \%$ \\
\hline Dislipidemia (n; \%) & $6 ; 27, .3 \%$ & $30 ; 66,7 \%$ \\
\hline Tabaquismo (n; \%) & $2 ; 9,1 \%$ & $19 ; 42,2 \%$ \\
IAM Previos (n; \%) & 0 & $5 ; 11,1 \%$ \\
& & \\
Al: Angina Inestable; IAM: Infarto Agudo de Miocardio; Otra: otra enfermedad \\
cardíaca.
\end{tabular}

Figura 1. Las MVEC/Exosomas purificadas desde plasma expresan CD9 y CD63 en pacientes con y sin SCA. Luego de filtrar el plasma por 0,2 $\mu \mathrm{m}$ se realizó el enriquecimiento de las MVEC por ExoQuick ${ }^{\circ}$. Se observa la expresión de CD9 (A) y CD63 (B), en fracción enriquecida de MVEC-Exosomas de plasma de 6 pacientes sin SCA y 6 pacientes con diagnóstico de SCA. Como control positivo, se emplearon exosomas obtenidos por ultracentrifugación (UC)
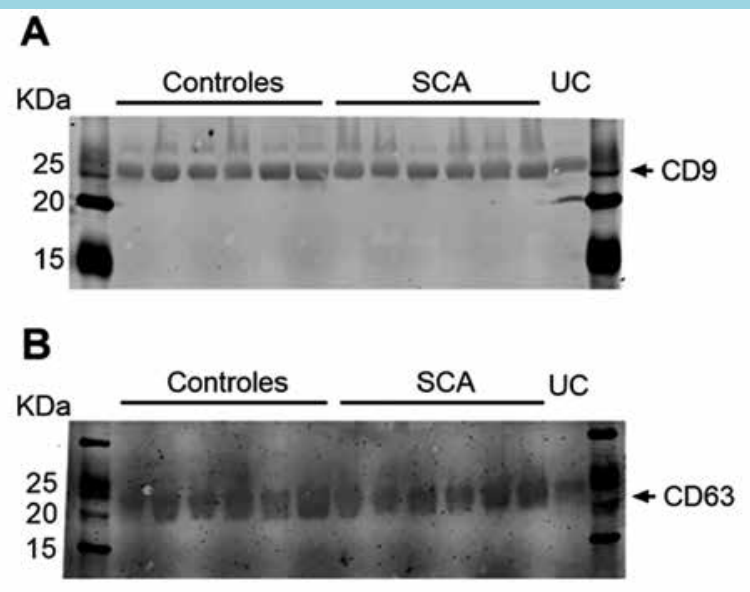

ción de MVEC ( $<5 \%$ de coeficiente de variación) desde plasma (datos no mostrados). La presencia de MVEC/ exosomas en la fracción enriquecida por ExoQuick se realizó mediante la determinación de la presencia de marcadores exosomales por Western blot de las proteínas CD9 y CD63 (Figura 1). Los datos muestran que tanto los individuos controles como los pacientes con SCA expresan niveles comparables de ambas tetraspaninas. Mediante NTA, se determinó la concentración y las poblaciones de distintos tamaños de las MVEC/Exosomas enriquecidas. La Figura 2 muestra la relación entre la concentración versus el tamaño de la MVEC/Exosomas de los pacientes controles y con SCA en el momento de ingreso a la unidad de Urgencia de la Clínica (0-2 h). Se observa que en los controles la concentración máxima es de 2,15 x109 MVEC/mL y corresponde a un tamaño de $150 \mathrm{~nm}$. Por otro lado, en los pacientes con SCA la concentración máxima observada es de 8,25 x109 MVEC/ $\mathrm{mL}$ y corresponde a un tamaño de $100 \mathrm{~nm}$., indicando que los pacientes con SCA experimentaron un aumento de la concentración de MVEC plasmática de menor tamaño en el momento de ingreso a urgencia (Figura 2).

Figura 2. El tamaño y concentración de las MVEC-Exosomas plasmáticas es modificado en los pacientes con SCA. Se muestra la relación entre la concentración y tamaño de MVEC/Exosomas en el grupo control (línea azul) y en pacientes con SCA (línea roja) al ingreso a la Clínica (0-2hrs).

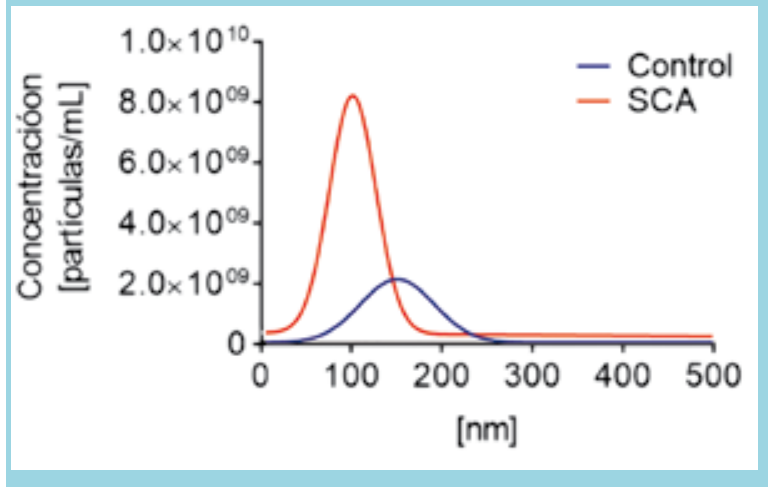

Debido a la gran variabilidad de los valores en el grupo SCA, los valores de troponina no fueron significativamente diferentes entre controles $(0,017+0,015)$ y sujetos con $\mathrm{SCA}(22,662+65,793 \mathrm{ng} / \mathrm{mL} \mathrm{p}=0.11)$. Además, los pacientes con SCA en muchos casos ingresaron con una troponina bajo el límite de cuantificación del ensayo (Figura 3A). La concentración plasmática promedio de las MVECs en los pacientes con SCA fue significativamente mayor que en el grupo control (Controles 0,92 x $10^{11} / \mathrm{mL}$; SCA $\left.6.6 \times 10^{11} / \mathrm{mL}, \mathrm{p}<0.0001\right)$. En promedio la concentración de MVEC fue 7,2 veces más alta en pacientes con SCA que en los controles al ingreso (Figura 3B), sugiriendo que las MVEC son liberadas desde el tejido cardíaco que está expuesto a algún tipo de daño en una fase previa a la necrosis, implicando una señal más temprana que la TnI. Debido a que el tama- 
ño de las MVEC/Exosomas disminuye en los pacientes con SCA, se analizaron distintas subpoblaciones de MVEC de plasma. El análisis mostró que la población de MVEC-Exosomas en el rango de 30-100 nm presentó la mayor diferencia entre los grupos estudiados, llegando a ser 14,4 veces superior en pacientes con SCA (Control: 0,178 versus SCA: $2,57 \times 10^{11} / \mathrm{mL}$ plasma, $\mathrm{p}<0.0001$ ) (Figura 3C).

Figura 3. Las MVEC-Exosomas plasmáticas poseen una mejor asociación con el SCA que la Troponina-I al ingreso a la urgencia. A) Concentración plasmática de Troponina-I (controles 0,017 \pm 0,0032 versus SCA 22,66 $\pm 65,79 \mathrm{ng} / \mathrm{mL}$ plasma) y B) Concentración de MVEC/Exosomas totales en el grupo control y con SCA de la primera muestra al ingreso a la Clínica (0-2 hrs). C) Concentración de MVEC/Exosomas de 30-100 nm en el grupo control y con SCA de la primera muestra (0-2 hrs) al ingreso a la Clínica. ( promedio + desviación estándar).

A

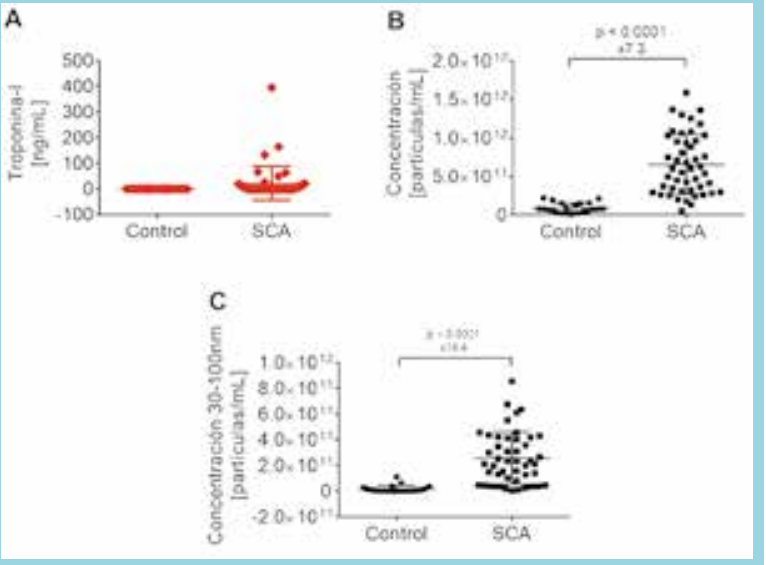

Debido a esta observación seleccionamos a aquellos pacientes que tuvieron una concentración de Troponina negativa al ingreso a la clínica y evolucionaron hacia una Troponina claramente positiva. En este grupo de pacientes analizamos la concentración de MVEC totales en controles y con SCA (evolución en 24 hrs) para establecer si el aumento de las MVEC fue una señal más precoz que la TnI en su asociación con SCA. Los datos muestran que el grupo de pacientes con SCA con TnI negativa al ingreso experimentaron un significativo incremento de la concentración promedio de TnI dentro de las 24 h de observación (Desde 0,35 $\pm 0,19 ; 38,59 \pm$ 25,$32 ; 60,52 \pm 38,46 \mathrm{ng} / \mathrm{mL}$ durante $0-2 ; 6-8$ y $10-14 \mathrm{~h}$, respectivamente). Por otro lado, la medición de MVEC de plasma demostró que en el momento del ingreso los pacientes con SCA tuvieron una concentración signifi- cativamente mayor que los controles $\left(6.28 \times 10^{11}\right.$ vs 0.33 x $\left.10^{11} \mathrm{MVEC} / \mathrm{mL}\right)$. El análisis del curso temporal estableció que la concentración promedio de las MVECs en SCA fue significativamente mayor que en el grupo control en todos los puntos observados, con una tenden-

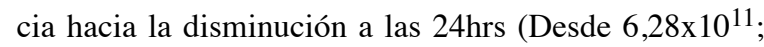
$4,45 \times 10^{11} ; 4,20 \times 10^{11} \mathrm{MVEC} / \mathrm{mL}$ durante $0-2 ; 6-8$ y 10 14h, respectivamente; Figura 4). Estos datos sugieren que las MVEC un diagnóstico de SCA más precoz que la troponina.

Figura 4. La concentración de las MVEC-Exosomas de 30-100 nm son elevadas al ingreso a urgencia en pacientes con Troponina negativa y que evolucionaron hacia una Tnl positiva en $24 \mathrm{~h}$. A) Concentración plasmática de Troponina I de pacientes controles (0-2 h) y con SCA (Curso temporal: 0-2 h, 1a; 6-8 h, 2a, 10-14 h, 3). B) Concentración de MVEC/Exosomas de la subpoblación de vesículas que está en el rango de 30-100nm presentes en plasma de pacientes controles (0-2hrs post ingreso) y con SCA (Curso temporal: 0-2hrs, $1^{\text {a }}$; 6-8hrs, $2^{\mathrm{a}} ; 10-14 \mathrm{hrs}, 3^{\mathrm{a}}$ ). ( Promedio \pm desviación estándar).

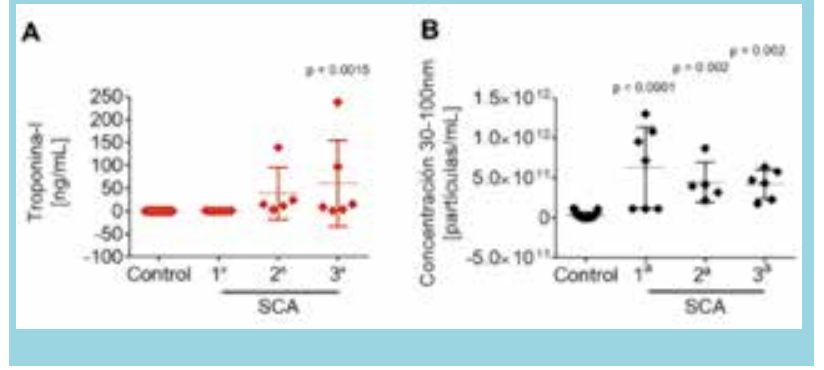

3. El incremento de MVEC-Exosomas en pacientes SCA es mayormente asociado a la condición coronaria. Como se muestra en la Tabla 1, los pacientes SCA presentaron un alto porcentaje de comorbilidades y factores de riesgo que podrían contribuir en la concentración de MVEC-Exosomas circulantes. Por esta razón, se estudió el efecto de diferentes comorbilidades factores de riesgo en los pacientes con SCA (Sin Comorbilidad, S/C; y Comorbilidad/factor de riesgo, COM). Como se muestra en la Figura 5, los pacientes con SCA poseen elevados niveles de MVEC/Exosoma en forma independiente de la presencia de Diabetes Mellitus, Hipertensión Arterial, dislipidemia y hábito de fumar y que la presencia de estos factores de riesgo no influye en la concentración de MVEC/Exosomas totales o las más pequeñas $(91-120 \mathrm{~nm})$.

\section{Discusión}

Este estudio proporciona evidencia nueva y muy rele- 
Figura 5. El aumento temprano de la concentración de MVEC-Exosomas en pacientes SCA no fue afectado por DM, HTA, DLP o TBQ. Los datos mostrados en la Figura 2, para pacientes SCA, fueron divididos en 2 sub grupos: Sin comorbilidad ( $\mathrm{S} / \mathrm{C}$ ) y con comorbilidad/factor de riesgo (COM), según lo mostrado en Tabla 1. A) Diabetes Mellitus Tipo 2 (DM2); B) Hipertensión Arterial (HTA); C) Dislipidemia (DLP); y D) Tabaquismo (TBQ). No se observaron diferencias significativas entre ambos sub grupos de pacientes SCA para MVEC-Exosomas totales ni para el rango 91-120 nm (paneles de la derecha (promedio \pm desviación estándar).
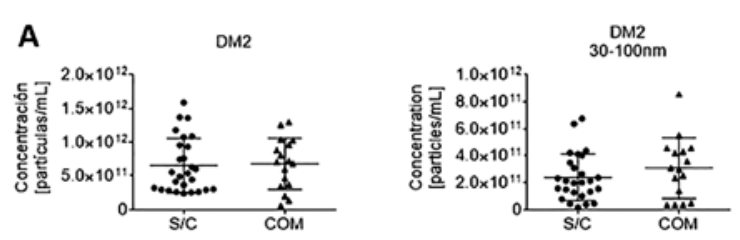

B
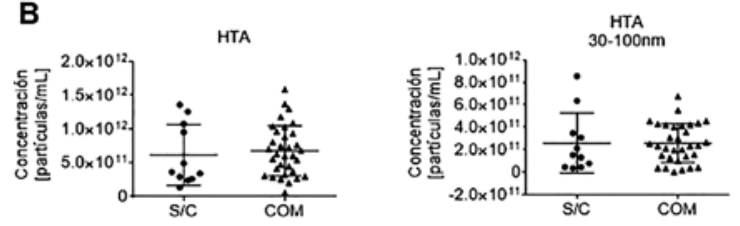

C
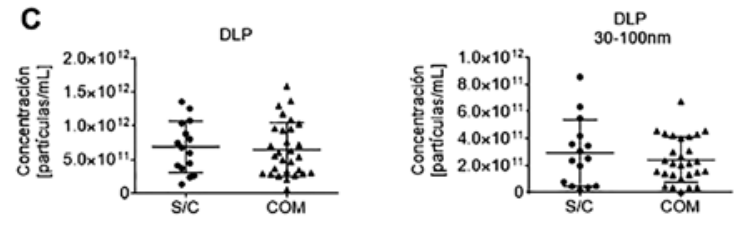

D
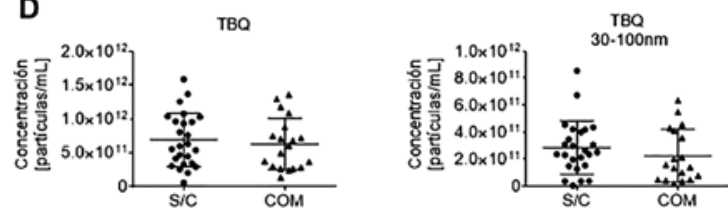

vante que demuestra que en las primeras $2 \mathrm{hrs}$ de iniciado un SCA, hay un incremento estadísticamente significativo de vesículas circulantes de tamaño 91-120nm, característico de la población de exosomas ${ }^{10,15}$ y que este aumento de MVEC ocurre antes que el incremento de TnI plasmática. La evidencia de la literatura demuestra que la principal causa del IAM es la falta de oxígeno suministrado por la circulación al tejido cardíaco ${ }^{1,3,26}$. En esta condición la elevación de TnI plasmática se debe a la destrucción de los cardiomiocitos y, por otro lado, como se ha descrito, las MVEC son secretados por células vivas como mecanismo de comunicación y respuesta frente a la agresión ${ }^{7,9}$. Es posible que de forma previa al IAM, las MVEC sean liberadas como agentes de super- vivencia desde los cardiomiocitos ${ }^{27,28}$. Basándose en los resultados expuestos proponemos que MVEC que deriven del corazón y circulen por el plasma, sirvan como indicadores precoces de riesgo de SCA.

Existen varios métodos para aislar MVEC/exosomas, dentro de los cuales existen kits comerciales. El "gold standard" es la ultracentrifugación, pero en el campo clínico su empleo no resulta de fácil implementación ${ }^{8,15}$. Otros métodos se basan en el uso de matriz de polímeros que permiten su fácil y rápida obtención. Este método, por lo demás, es aplicable en clínica debido a su bajo costo, al reducido tiempo de realización y no requiere personal especializado ${ }^{8,15-19}$. En nuestro laboratorio, hemos implementado la extracción de MVECs mediante el uso de ExoQuick®, el cual purifica una fracción enriquecida de exosomas, que además se recomienda para la caracterización de diversas moléculas como proteínas y RNA ${ }^{10,16-18,20,21}$. Mediante este protocolo se pudo establecer que este método permite la purificación de MVEC/exosomas a partir de un volumen menor de plasma $(250 \mu \mathrm{L})$ en comparación con la ultracentrifugación (que requiere al menos $5 \mathrm{~mL}$ ), permitiendo no sólo una optimización de la muestra del paciente, sino también en el tiempo de purificación y la dependencia de equipamiento de costo elevado y personal calificado. Actualmente, la caracterización de marcadores exosomales, sobre la fracción enriquecida de MVECs desde plasma, incluye expresión de tetraspaninas CD9/CD639, 22, 19 y estudios de tamaño mediante Nanoparticle Tracking Assay (NTA, Nanosight). ${ }^{8,15,23,24}$

Nuestros datos permiten establecer que el enriquecimiento de MVEC desde plasma humano por un método comercial permite su caracterización mediante clásicos marcadores CD9 y $\mathrm{CD}^{9} 3^{9}, 19,22$.

En una publicación reciente se describe una aproximación similar a la nuestra, pero en pacientes derivados a cirugía cardiovascular. Observaron que poblaciones de MVEC de pequeño tamaño (61-90 nm y 91-120 nm) estaban elevadas en pacientes que recibieron cirugías de injertos de bypass coronario y que este efecto se correlacionaba con el incremento de Troponina cardíaca ${ }^{12}$. Sin embargo, en este estudio la cirugía cardíaca per se puede elevar los niveles de vesículas secretadas como respuesta al daño, o bien, por ruptura celular. Nuestro modelo, en cambio, evalúa un escenario fisiológico en que la única condición anómala es la enfermedad coronaria del paciente. Respecto de esto, demostramos que las comorbilidades relacionadas con eventos coronarios y daño cardíaco ${ }^{29}$ de los pacientes con SCA reclutados 
para este estudio, no afectaron los niveles de MVEC totales o las más pequeñas (91-120nm). En otras palabras, el incremento temprano de MVEC-Exosomas se produjo porque los pacientes tenían daño/amenaza cardíaca, generado probablemente por isquemia.

El estado de esta investigación sugiere que el aumento de la concentración de las MVEC de alrededor de 100nm aumenta durante el SCA en forma previa a la Troponina I y que DM, HTA, DLP o Tabaquismo no influyen, sugiriendo que las MVEC son una fuente de biomarcadores de enfermedades cardiovasculares.

Nuestro próximo paso es profundizar en el concepto de que MVEC-Exosomas son marcadores riesgo y por tanto previo a la necrosis celular. Un sistema de alarma y comunicación que se determinaría por el contenido microvesicular, los cuales incluyen proteínas y ácidos nucleicos (DNA, RNA), cuyo destino y efectos aún no se conocen. Para ello comenzamos a reclutar pacientes con pruebas de provocación de isquemia positivas (Test de esfuerzo, ecocardiograma de estrés, cintigrafía miocárdica) a los cuales se les medirá de manera seriada los niveles de MVEC-Exosomas circulantes en el momento de la realización del test y se efectuará un extenso análisis de su contenido molecular. 


\section{Referencias}

1. MOZAFFARIAN D, BENJAMIN E, GO A, ARNETT D, BLAHA M, CUSHMAN M, et al. Executive Summary: Heart Disease and Stroke Statistics--2016 Update: A Report From the American Heart Association.. Circulation.2016; 133:447-454.

2. MINISTERIO DE SALUD, SANTIAGO, M. Guía clínica infarto agudo del miocrdio con supradesnivel del segmento ST. 2010.

3. AHMED A, SHANKAR KJ, EFTEKHARI H, MUNIR MS, ROBERTSON J, BREWER A, et al. Silent myocardial ischemia: Current perspectives and future directions. Exp. Clin. Cardiol. 2007; 12:189-96.

4. CHAPELlE J, DUBOIS B, BOVY C, ALDENHOFF MC, GIELEN J, RORIVE G. Comparison of plasma cardiac tropo$\operatorname{nins} \mathrm{T}$ and $\mathrm{I}$ in chronically hemodialyzed patients in relation to cardiac status and age. Clin. Chem. Lab. Med. 2002; 40: 240-5.

5. SACHDEVA A, PAUL B. Newer cardiac biomarkers in myocardial necrosis. J. Assoc. Physicians India 2014; 62: 83-4.

6. SAIKI A, IWASE M, TAKEICHI Y, UMEDA H, ISHIKI R, INAGAKI H, et al. Diversity of the elevation of serum cardiac troponin I levels in patients during their first visit to the emergency room. Circ. J. 2007; 71: 1458-62.

7. COSME J, LIU P, GRAMOLINI A. The cardiovascular exosome: Current perspectives and potential. Proteomics 2013; 13: $1654-1659$.

8. SÁENZ-CUESTA M, ARBELAIZ A, OREGI A, IRIZAR H, OSORIO-QUEREJETA I, MUÑOZ-CULLA M, et al. Methods for extracellular vesicles isolation in a hospital setting. Front. Immunol.2015; 6: 50.

9. YUAN M, MAGHSOUDI T, WANG T. Exosomes mediate the intercellular communication after myocardial infarction. Int. J. Med. Sci. 2016; 13: 113-116.

10. WONG WY, LEE MM, CHAN BD, KAM RK, ZHANG G, LU AP, et al. Proteomic profiling of dextran sulfate sodium-induced acute ulcerative colitis mice serum exosomes and their im- munomodulatory impact on macrophages. Proteomics . 2016; 16: $1131-45$.

11. DAVIDSON SM, TAKOV K, YELLON DM. Exosomes and Cardiovascular Protection..Cardiovasc Drugs Ther. 2017; 31: $77-86$.

12. EMANUELI C, SHEARN AI, LAFTAH A, FIORENTINO F, REEVES BC, BELTRAMI C,et al. Coronary Artery-BypassGraft Surgery Increases the Plasma Concentration of Exosomes Carrying a Cargo of Cardiac MicroRNAs: An Example of Exosome Trafficking Out of the Human Heart with Potential for Cardiac Biomarker Discovery. PLoS One 2016; 11: e0154274.

13. PIRONTI G, STRACHAN RT, ABRAHAM D, MON-WEI YU S, CHEN M, CHEN W, et al. Circulating Exosomes Induced by Cardiac Pressure Overload Contain Functional Angiotensin II Type 1 Receptors. Circulation 2015; 131: 2120-30.

14. GUPTA S, KNOWLTON A. HSP60 trafficking in adult cardiac myocytes: role of the exosomal pathway. Am. J. Physiol. 2007; 292: H3052-6.

15. EMILY ZERINGER, MU LI, TIM BARTA, JEOFFREY SCHAGEMAN, KETIL WINTHER PEDERSEN, AXL NEURAUTER, et al. Methods for the extraction and RNA profiling of exosomes. World J Methodol. 2013; 3: 11-18.

16. DI PIETRO C. Exosome-mediated communication in the ovarian follicle. J. Assist. Reprod. Genet. 2016; 33: 303-11.

17. WANG X, HUANG W, LIU G, CAI W, MILLARD RW, WANG Y, et al. Cardiomyocytes mediate anti-angiogenesis in type 2 diabetic rats through the exosomal transfer of miR-320 into endothelial cells.. J. Mol. Cell. Cardiol. 2014; 74: 139-50.

18. FENG, Y, HUANG W, WANI M, YU X, ASHRAF M. Ischemic preconditioning potentiates the protective effect of stem cells through secretion of exosomes by targeting Mecp2 via miR-22. PLoS One 2014; 9: 1-8.

19. KOWAL J, ARRAS G, COLOMBO M, JOUVE M, MORATH JP, PRIMDAL-BENGTSON B, et al. Proteomic comparison 
defines novel markers to characterize heterogeneous populations of extracellular vesicle subtypes. Proc. Natl. Acad. Sci. U. S. A. 2016; 113: E968-77.

20. MATSUMOTO S1, SAKATA Y, SUNA S, NAKATANI D, USAMI M, HARA M, et al. Circulating p53-responsive microRNAs are predictive indicators of heart failure after acute myocardial infarction. Circ. Res. 2013;113: 322-6.

21. LI J, ROHAILLA S, GELBER N, RUTKA J, SABAH N, GLADSTONE R, et al. MicroRNA-144 is a circulating effector of remote ischemic preconditioning. Basic Res. Cardiol. 2014; 109: $1-15$.

22. LAI, R. C. et al. Exosome secreted by MSC reduces myocardial ischemia/reperfusion injury. Stem Cell Res. 2010; 4: 214-222.

23. SAARI H, LÁZARO-IBÁÑEZ E, VIITALA T, VUORIMAA-LAUKKANEN E, SILJANDER P, YLIPERTTULA M. Microvesicle- and exosome-mediated drug delivery enhances the cytotoxicity of Paclitaxel in autologous prostate cancer cells. J. Control. Release 2015; 220: 727-737.
24. AILAWADI S, WANG X, GU H, FAN GC. Pathologic function and therapeutic potential of exosomes in cardiovascular disease. Biochim. Biophys. Acta - Mol. Basis Dis. 2015; 1852: 1-11.

25. LOH TP et al. Original papers. 2016;26: 233-239.

26. GRAMLEY F, LORENZEN J, JEDAMZIK B, GATTER K, KOELLENSPERGER E, MUNZEL T,et al. Atrial fibrillation is associated with cardiac hypoxia. Cardiovasc. Pathol. 2010; 19: $102-111$.

27. CERVIO E, BARILE L, MOCCETTI T, VASSALLI G. Exosomes for Intramyocardial Intercellular Communication. Stem Cells Int. 2015, 482171.

28. IBRAHIM A, MARB E, MARBÁN E. EXOSOMES: Fundamental Biology and Roles in Cardiovascular Physiology. Annu. Rev. Physiol. 2016;78:67-83.

29. AGHDAM MRF, VODOVNIK A, SUND BS. Sudden death associated with silent myocardial infarction in a 35-year-old man: a case report. J. Med. Case Rep. 2016; 10: 46. 